\title{
Late vitreomacular traction in toxoplasma retinochoroiditis resolved by vitrectomy
}

This article was published in the following Dove Press journal:

Clinical Ophthalmology

6 September 2013

Number of times this article has been viewed

\section{Giuseppe Scarpa \\ Stefano Fabris \\ Marco Di Gregorio \\ Francesca Urban}

Ophthalmic Department Regional Hospital, Ca' Foncello Piazza

Ospedale I, Treviso, Italy
Correspondence: Marco Di Gregorio Ophthalmic Department Regional Hospital, Ca' Foncello Piazza Ospedale I, 3I I00 Treviso, Italy

Tel +3934 804l 0655

Fax +39042232 2509

Email marco.digregoriomd@gmail.com

\begin{abstract}
This paper reports a case of late vitreomacular traction in a young patient secondary to toxoplasma retinochoroiditis resolved by vitrectomy. A 17-year-old female with chronic inflammatory bowel disease developed severe vitreomacular traction 8 months after resolution of ocular toxoplasmosis with medical therapy. Best-corrected visual acuity, full ophthalmic slitlamp examination, colour fundus photography, spectral domain optical coherence tomography, and fluorescein angiography were performed. The patient underwent vitrectomy with removal of the clinically evident posterior hyaloid. Vitrectomy was rapidly successful in resolving the vitreomacular traction, with full recovery in best-corrected visual acuity of 20/20. Vitreoretinal traction in patients with previous toxoplasma retinochoroiditis may appear several months after resolution of the inflammatory condition. We suggest observing carefully for possible development of late vitreoretinal traction during follow-up of such patients.
\end{abstract}

Keywords: ocular toxoplasmosis, toxoplasma retinochoroiditis, vitreomacular traction, vitrectomy, inflammatory bowel disease

\section{Introduction}

Ocular toxoplasmosis is the major cause of posterior uveitis in European populations. The infection usually induces retinochoroiditis, frequently localized at the posterior pole, with destruction of the inner retinal structures, the retinal pigment epithelium, and the choroid. Vitreous haze and cells can sometimes be observed in the vitreous compartment overlying the site of inflammation, but usually clear completely with no vitreoretinal consequences as the inflammation resolves. We report an unusual case in which typical toxoplasma retinochoroiditis induced late progressive vitreomacular traction. Vitrectomy with removal of the adherent posterior hyaloid was successful in completely restoring visual acuity.

\section{Case report}

A 17-year-old female presented to our department with progressive blurring of vision in the left eye. She had been diagnosed with chronic inflammatory bowel disease 3 years earlier and was in remission on azathioprine and mesalazine. The patient underwent best-corrected visual acuity testing, full ophthalmic slit-lamp examination of the anterior and posterior segments, spectral-domain optical coherence tomography (SD-OCT), and fluorescein angiography at baseline. A complete serologic search was performed to detect an infectious cause, and active toxoplasma retinochoroiditis was diagnosed. A classic treatment regimen of pyrimethamine and sulfadiazine plus steroids was started. 
During the ensuing observation period, the patient was evaluated every 2 months and underwent the same examinations performed at baseline except for fluorescein angiography. When the clinical course suddenly evolved toward a significant vitreoretinal traction due to retraction of the retinal scar, pars plana vitrectomy was considered. After written informed consent was obtained from the patient's parents, a three-port pars plana vitrectomy was performed with a 23-gauge transconjunctival approach. The posterior hyaloid was removed intraoperatively using forceps and no attempt was made to remove the inner limiting membrane.

On presentation, visual acuity was 20/20 in the right eye and $1 / 20$ in the left eye, with a mild relative afferent pupillary defect in the second one. No other neurologic signs or symptoms were detected. Anterior segment examination in both eyes was unremarkable and intraocular pressure was $16 \mathrm{mmHg}$. On biomicroscopic examination, the vitreous of the right eye was clear whereas the left eye exhibited mild inflammation. Fundus examination of the left eye showed a whitish yellow inflammatory lesion temporal to the macula surrounded by retinal edema with foveal involvement (Figure 1A). The lesion was apparently located under the retinal pigment epithelium and there was mild dilation of the retinal vessels of the macula. No other retinal lesion was detected in the peripheral retina. Fluorescein angiography showed early hypofluorescence in the central part of the lesion with progressive hyperfluorescence of the margins due to leakage and pooling of the dye (Figure 1B and C).

A serologic search for Toxoplasma gondii infection was positive for immunoglobulin $\mathrm{G}(55.4 \mathrm{UI} / \mathrm{mL}$, reference value $<10 \mathrm{UI} / \mathrm{mL}$ ), whereas immunoglobulin $\mathrm{M}$ levels were normal, indicating prior exposure to Toxoplasma. A syphilis screen and other tests, including for toxocariasis, and rheumatologic tests were all negative. A presumptive diagnosis of toxoplasma retinochoroiditis with macular involvement was made, and a regimen of pyrimethamine, sulfadiazine, and steroids was started.

Prompt reduction of the retinal edema was followed by gradual flattening and pigmentation of the lesion, with a progressive increase in visual acuity from 20/200 after 4 days

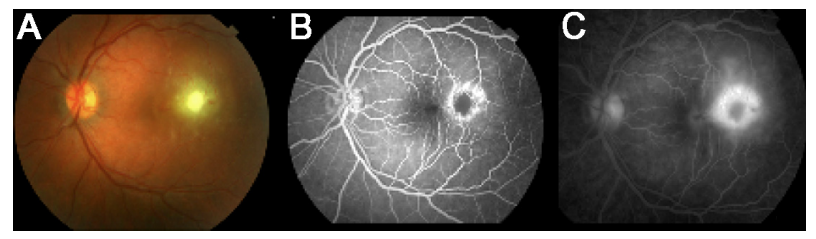

Figure I Color fundus photography (A) and fluorescein angiography (B and $\mathbf{C}$ ) of the left eye. to $20 / 40$ after 20 days. Medical therapy was stopped after 45 days. In the following months, no relapse of retinochoroidal inflammation or vitreitis was observed, and visual acuity remained stable during the first 6 months of observation. At month 2, a thickening of the vitreoretinal interface, interpreted as an initial epiretinal membrane, developed above the macula, without significant vitreoretinal traction or macular edema detectable by SD-OCT. However, this finding prevented complete recovery of visual function.

By the 8-month follow-up visit, visual acuity had declined to 20/200, with an increase in metamorphopsia. Clinical observation and SD-OCT revealed hyper-reflectivity of the vitreoretinal interface, suggesting a thickening of the posterior hyaloid strongly adherent to several areas of the macula, inducing elevation and marked distortion of the entire macular area (Figure 2A). These ocular clinical findings were suggestive of an inactive scar-like lesion without any inflammatory reaction in the vitreous and anterior chamber, but complicated by significant vitreoretinal traction.

After written informed consent was obtained from the patient's parents, a 23-G pars plana vitrectomy was performed. The posterior hyaloid was removed intraoperatively using forceps, without difficulty. After having accurately verified that no epiretinal membrane was detectable in the entire macular area, no attempt was made to remove the inner limiting membrane. Vitrectomy was completed without complication in a total surgical time of 20 minutes. Visual acuity improved soon after surgery and reached 40/60 after 10 days. The macula remained flat and free of any signs of traction in the following 10 months, with stabilization of visual acuity to 20/20 (Figure 2B).

\section{Discussion}

Toxoplasma eye infection usually affects the intraretinal structures and choroid of the posterior pole, producing

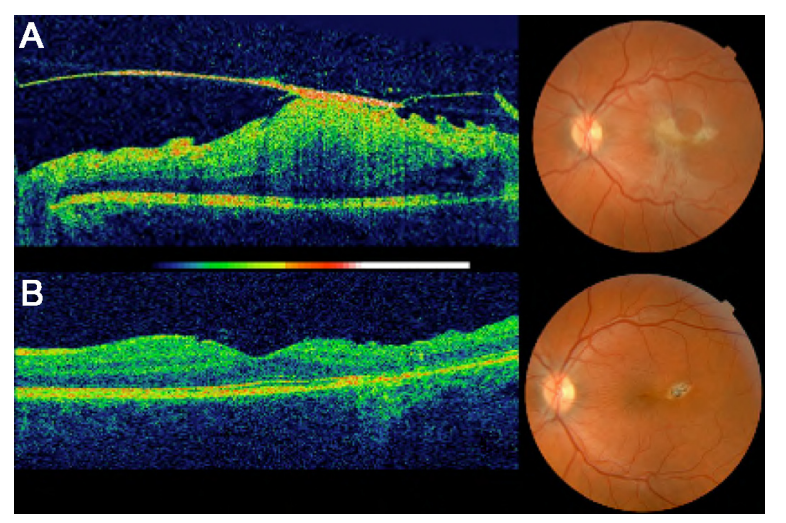

Figure 2 Spectral domain optical coherence tomography B-scan images before (A) and 2 months after surgery (B). 
acute localized foci of necrotizing inflammation, resulting in localized destruction of the retinochoroidal tissue. Inflammatory processes involving neighboring structures produce alterations in the retinal vessels, leading to breakdown of the blood-retinal barrier. Retinal edema, venous beading, and vitreous flare can then develop. The inflammatory process usually disappears with resolution at the primary infection site. Therefore, the mainstay of therapy is to treat the infection with drugs active against the infective agent and to protect the delicate surrounding retinal structures from the effects of reactive inflammation. Due to the severity of macular localization, this condition demands prompt and aggressive combined therapy. In contrast, vitreous involvement is generally considered to be a more benign condition, with the vitreous inflammatory reaction usually resolving completely with no functional consequences.

Posterior hyaloid thickening and adhesion to the sites of active inflammation with vitreomacular traction have recently been demonstrated by both spectral and time domain OCT in ocular toxoplasmosis, but relief of the vitreomacular traction with complete detachment of the posterior hyaloid usually occurs spontaneously in the healing phase in many patients. ${ }^{1-3}$ However, some authors have reported cases in which ocular toxoplasmosis is associated with early-onset vitreoretinal complications, including epiretinal membrane formation and rhegmatogenous and tractional retinal detachment. ${ }^{4-7}$

In the case reported here, the primary infection was treated promptly with a satisfactory medical response, but significant vitreomacular traction developed 8 months following completion of therapy. This late vitreous contraction was probably triggered by subclinical vitreous inflammation and aggravated by the underlying inflammatory bowel disease. It is possible that tissue remodeling and fibrosis of the toxoplasmic retinal lesion was enhanced by alteration of the biological mediators and pathways similarly implicated in the pathogenesis of the patient's gastrointestinal disease. The functional importance of such mechanical damage to the macular structures is demonstrated by the dramatic visual improvement observed immediately after relief of the traction by vitrectomy.

This case report confirms that toxoplasma retinochoroiditis, like many other inflammatory diseases associated with vitreitis, may cause a tough vitreoretinal traction; what is relevant in this case is the time of presentation of the traction itself, ie, its appearance many months after resolution of the inflammatory condition. Therefore, we suggest that care should be taken during follow-up with ophthalmoscopy and SD-OCT, and that the patient should be counseled that a recurrence or change in vision may indicate the development of vitreoretinal traction, particularly if there is a history of toxoplasma retinochoroiditis.

\section{Disclosure}

The authors report no conflicts of interest in this work.

\section{References}

1. Orefice JL, Costa RA, Orefice F, Campos W, da Costa-Lima D, Scott IU. Vitreoretinal morphology in active ocular toxoplasmosis: a prospective study by optical coherence tomography. Br J Ophthalmol. 2007;91:773-780.

2. Diniz B, Regatieri C, Andrade R, Maia A. Evaluation of spectral domain and time domain optical coherence tomography findings in toxoplasmic retinochoroiditis. Clin Ophthalmol. 2011;5:645-650.

3. Cho DY, Nam W. A case of ocular toxoplasmosis imaged with spectral domain optical coherence topography. Korean J Ophthalmol. 2012;26: $58-60$.

4. Rothova A. Ocular manifestations of toxoplasmosis. Curr Opin Ophthalmol. 2003;14:384-388.

5. Smith JR, Cunningham ET. Atypical presentations of ocular toxoplasmosis. Curr Opin Ophthalmol. 2002;13:387-392.

6. Adàn A, Giralt J, Alvarez G, et al. Pars plana vitrectomy for vitreoretinal complications of ocular toxoplasmosis. Eur J Ophthalmol. 2009;19: 1039-1043.

7. Papadopoulou DN, Petropoulos IK, Mangioris G, Pharmakakis NM, Pournaras CJ. Pars plana vitrectomy in the treatment of severe complicated toxoplasmic retinochoroiditis. Eur J Ophthalmol. 2010;21:83-88.
Clinical Ophthalmology

\section{Publish your work in this journal}

Clinical Ophthalmology is an international, peer-reviewed journal covering all subspecialties within ophthalmology. Key topics include: Optometry; Visual science; Pharmacology and drug therapy in eye diseases; Basic Sciences; Primary and Secondary eye care; Patient Safety and Quality of Care Improvements. This journal is indexed on

Submit your manuscript here: http://www.dovepress.com/clinical-ophthalmology-journal

\section{Dovepress}

PubMed Central and CAS, and is the official journal of The Society of Clinical Ophthalmology (SCO). The manuscript management system is completely online and includes a very quick and fair peer-review system, which is all easy to use. Visit http://www.dovepress.com/ testimonials.php to read real quotes from published authors. 